Supporting information

\title{
Olefin Cross-metathesis as a Tool in Natural Products Degradation. The Stereochemistry of $(+)$-Falcarindiol.
}

\author{
Anokha S. Ratnayake and Thomas Hemscheidt* \\ Department of Chemistry, University of Hawaii, 2545 McCarthy Mall, Honolulu, HI 96822, USA
}


( \pm )-5-Trimethylsilylpent-1-en-4-yne-3-ol 3.

To a solution of (trimethylsilyl)acetylene $(1.04 \mathrm{~g}, 1.5 \mathrm{~mL}, 10.6 \mathrm{mmol})$ in dry THF (42 mL) at $78{ }^{\circ} \mathrm{C}$ was added dropwise a solution of n-butyllithium (2.4 $\mathrm{M}$ in hexanes, $\left.4.42 \mathrm{~mL}, 10.6 \mathrm{mmol}\right)$ under a $\mathrm{N}_{2}$ atmosphere. After the addition was complete, the solution was allowed to warm to $60{ }^{\circ} \mathrm{C}$ over a period of $15-30 \mathrm{~min}$, at which point an ice-cold solution of acrolein $(0.71 \mathrm{~g}, 0.85$ $\mathrm{mL}, 12.7 \mathrm{mmol})$ in THF (25 mL) was introduced slowly via a cannula. The resulting mixture was allowed to gradually warm to $0{ }^{\circ} \mathrm{C}$ over a period of $1.5 \mathrm{~h}$. After stirring for an additional $1 \mathrm{~h}$ at room temperature, the mixture was quenched with cold satd. $\mathrm{NH}_{4} \mathrm{Cl}$. The aqueous phase was extracted with $\mathrm{Et}_{2} \mathrm{O}(\times 3)$, dried $\left(\mathrm{MgSO}_{4}\right)$ and concentrated to dryness in vacuo. The resulting crude oil was purified by Kugelrohr distillation (bp $85-90{ }^{\circ} \mathrm{C} / 20 \mathrm{mmHg}$ ) to give $1.63 \mathrm{~g}(95 \%$ yield) of pure racemic allyl alcohol $( \pm$ )-3 as a colorless liquid. FTIR (film) 3355 (br), 2175, 1640, 1405, 1250, 1115, 1030, 985, 845, $760 \mathrm{~cm}^{-1}$; ${ }^{1} \mathrm{H}$ NMR $\left(300 \mathrm{MHz}, \mathrm{CDCl}_{3}\right) \delta 5.94(\mathrm{ddd}, \mathrm{J}=$ $17.1,10.2,5.4 \mathrm{~Hz}, 1 \mathrm{H}), 5.44(\mathrm{dd}, \mathrm{J}=17.1,0.9 \mathrm{~Hz}, 1 \mathrm{H}), 5.19(\mathrm{dd}, \mathrm{J}=10.2,0.9 \mathrm{~Hz}, 1 \mathrm{H}), 4.85($ brd, $\mathrm{J}=5.4 \mathrm{~Hz}, 1 \mathrm{H}), 2.60(b r \mathrm{~s}, 1 \mathrm{H}), 0.16(\mathrm{~s}, 9 \mathrm{H}) ;{ }^{13} \mathrm{C} \mathrm{NMR}\left(75 \mathrm{MHz}, \mathrm{CDCl}_{3}\right) \delta 136.9,116.7,104.3$, 91.2, 63.6, $-0.03(3 \mathrm{C})$.

(+)-5-Trimethylsilylpent-1-en-4-yne-3-ol (S)-3

To a well-stirred suspension of lipase (Pseudomonas fluorescens, $0.03 \mathrm{~g}, 0.15$ mass equiv), ground, activated $4-\AA$ molecular sieves $(0.22 \mathrm{~g}, 1.0$ mass equiv) and racemic allyl alcohol ( \pm )-3 $(0.22 \mathrm{~g}, 1.45 \mathrm{mmol})$ in dry hexane $(12 \mathrm{~mL})$ was added vinyl acetate $(0.53 \mathrm{~mL}, 5.8 \mathrm{mmol})$ in one portion. The suspension was allowed to stir under $\mathrm{N}_{2}$, at room temperature for $21 \mathrm{~h}$, and the course of the reaction was monitored by ${ }^{1} \mathrm{H}$ NMR analysis. At the end of this period, the mixture was filtered through a pad of Celite, and evaporated to dryness under reduced pressure. The crude product mixture following flash chromatography gave $(R)$-acetate (eluent: 3\% $\mathrm{Et}_{2} \mathrm{O} /$ hexane) $0.20 \mathrm{~g}$ (35\% yield, ee $>98 \%$ ) and alcohol $(S)-3$ ((eluent: $10 \% \mathrm{Et}_{2} \mathrm{O} /$ hexane) $0.16 \mathrm{~g}$ $(35 \%$ yield, ee $>98 \%)$ as colorless oils: $(S)-3 \geq 95 \%$ ee; $(\alpha)_{\mathrm{D}}{ }^{23} 38$ (c 2.0, $\left.\mathrm{CHCl}_{3}\right)$; for spectroscopic data see $( \pm)-3$.

(+)-5-bromopent-1-en-4-yne-3-ol $(S)-4$.

A solution of alcohol $(S)-3(0.05 \mathrm{~g}, 0.32 \mathrm{mmol})$ in acetone $(2 \mathrm{~mL})$ was treated with $\mathrm{N}$ bromosuccinimide $(0.07 \mathrm{~g}, 0.39 \mathrm{mmol})$ and catalytic powdered $\mathrm{AgNO}_{3}(0.004 \mathrm{~g}, 0.02 \mathrm{mmol})$. The reaction mixture was wrapped with aluminum foil to exclude light and stirred at room temperature for $3 \mathrm{~h}$. The mixture was diluted with cold water and extracted with $\mathrm{Et}_{2} \mathrm{O}(\times 3)$. The combined organic layers were dried $\left(\mathrm{MgSO}_{4}\right)$, concentrated to dryness in vacuo and subjected to flash column chromatography (silica gel, $10 \% \mathrm{Et}_{2} \mathrm{O}$-hexane) to afford $0.03 \mathrm{~g}$ (55\% yield) of bromo acetylene $(S)-\mathbf{4}$ as a clear liquid: $(\alpha)_{\mathrm{D}}{ }^{25} 38$ (c 1.9, $\mathrm{CHCl}_{3}$ ); FTIR (film) 3380 (br), 2215, 1640, 1405, 1265, 1120, 1015, 985, 935, 885, 800, $725 \mathrm{~cm}^{-1} ;{ }^{1} \mathrm{H}$ NMR $\left(300 \mathrm{MHz}, \mathrm{CDCl}_{3}\right) \delta 5.95$ (ddd, J=17.1, 9.9, $5.4 \mathrm{~Hz}, 1 \mathrm{H}), 5.46(\mathrm{ddd}, \mathrm{J}=17.1,0.9,0.9 \mathrm{~Hz}, 1 \mathrm{H}), 5.25$ (ddd, J= 9.9, 0.9, 0.9 $\mathrm{Hz}, 1 \mathrm{H}), 4.90(\mathrm{ddd}, \mathrm{J}=6.3,5.4,0.9 \mathrm{~Hz}, 1 \mathrm{H}), 2.15(\mathrm{~d}, \mathrm{~J}=6.3 \mathrm{~Hz}, 1 \mathrm{H}) ;{ }^{13} \mathrm{C} \mathrm{NMR}(75 \mathrm{MHz}$, $\left.\mathrm{CDCl}_{3}\right) \delta 136.4,117.2,79.0,64.2,47.2$.

(S,S)-1,9-decadiene-4,6-diyne-3,8-diol 2.

To an ice-cold solution of $70 \%$ aqueous $\mathrm{EtNH}_{2}(0.060 \mathrm{~mL})$ and catalytic $\mathrm{CuCl}(0.002 \mathrm{~g}, 0.02$ $\mathrm{mmol})$ in $\mathrm{MeOH}(0.074 \mathrm{~mL}$, degassed with $\mathrm{Ar})$ was added $\mathrm{NH}_{2} \mathrm{OH} . \mathrm{HCl}(0.007 \mathrm{~g}, 0.11 \mathrm{mmol})$ 
dissolved in water $(0.026 \mathrm{~mL})$. To the above mixture at $0{ }^{\circ} \mathrm{C}$ was added a solution of bromo acetylene $(S)-4(0.052 \mathrm{~g}, 0.32 \mathrm{mmol})$ in $\mathrm{MeOH}(0.65 \mathrm{~mL})$. After the addition was complete, the reaction mixture was removed from the ice-bath and stirred at room temperature overnight. The solution was diluted with cold water and extracted with $\mathrm{Et}_{2} \mathrm{O}$. The organic layers were dried $\left(\mathrm{MgSO}_{4}\right)$, concentrated under reduced pressure and the resulting crude residue was purified on silica gel (20\%-30\% EtOAc-hexane) to yield $0.011 \mathrm{~g}$ (40\% yield) of symmetrical dimer $(S, S)-2$ as a white crystalline solid: $[\alpha]_{\mathrm{D}}{ }^{24} 102\left(\mathrm{c} 0.38, \mathrm{CHCl}_{3}\right)$; mp 77-78 ${ }^{\circ} \mathrm{C}$; FTIR (film) 3320 (br), 2355, 2145, 1635, 1405, 1260, 1115, 1015, 985, 935, $865 \mathrm{~cm}^{-1} ;{ }^{1} \mathrm{H}$ NMR $\left(300 \mathrm{MHz}, \mathrm{CDCl}_{3}\right) \delta$ 5.95 (ddd, J=17.1, 10.2, $5.4 \mathrm{~Hz}, 2 \mathrm{H}), 5.48$ (ddd, J=17.1, 0.9, 0.9 Hz, 2H), 5.27 (ddd, J= 10.2, 0.9, $0.9 \mathrm{~Hz}, 2 \mathrm{H}), 4.95$ (brm, 2H), $2.03(\mathrm{~d}, \mathrm{~J}=4.8 \mathrm{~Hz}, 2 \mathrm{H}) ;{ }^{13} \mathrm{C} \mathrm{NMR}\left(75 \mathrm{MHz}, \mathrm{CDCl}_{3}\right) \delta 135.9$ (2C), 117.7 (2C), $78.6(2 \mathrm{C}), 70.3(2 \mathrm{C}), 63.7$ (2C).

Degradation of (+)-1 to 2 .

A solution of second-generation Grubbs' catalyst $(0.011 \mathrm{~g}, 0.013 \mathrm{mmol}, 10 \mathrm{~mol} \%)$ in degassed anhydrous $\mathrm{CH}_{2} \mathrm{Cl}_{2}(45.5 \mathrm{~mL})$ at room temperature was stirred vigorously under an atmosphere of ethylene gas. After bubbling a steady stream of ethylene gas for $15 \mathrm{~min}$, a solution of (+)-1 $(0.035 \mathrm{~g}, 0.13 \mathrm{mmol})$ in degassed $\mathrm{CH}_{2} \mathrm{Cl}_{2}(70 \mathrm{~mL})$ under ethylene gas was added slowly via a cannula to the above reaction flask at room temperature. Progress of the reaction was monitored by TLC analysis. After stirring overnight (16 h), the solvent was removed in vacuo and the residue was purified by flash column chromatography (silica gel, 20-30\% EtOAc-hexane) to afford the symmetrical dimer 2-(meso) as a slightly brown solid. The crude solid was recrystallized in $\mathrm{Et}_{2} \mathrm{O}$-hexane to give $0.018 \mathrm{~g}$ ( $81 \%$ yield) of pure product as white crystalline material: $[\alpha]_{\mathrm{D}}{ }^{23} 5$ (c $0.7, \mathrm{CHCl}_{3}$ ); mp 49-51 ${ }^{\circ} \mathrm{C}$; FTIR (film) 3300 (br), 2360, 2145, 1640, 1400, 1325, 1260, 1115, 1015, 985, 930, $865 \mathrm{~cm}^{-1} ;{ }^{1} \mathrm{H}$ NMR $\left(500 \mathrm{MHz}, \mathrm{CDCl}_{3}\right) \delta 5.94$ (ddd, J= 17.0, 10.1, $5.4 \mathrm{~Hz}, 2 \mathrm{H}), 5.48$ (d, J=17.0 Hz, 2H), 5.27 (d, J=10.1 Hz, 2H), 4.95 (d, J= 5.4 Hz, 2H), 2.36 (brs, 2H); ${ }^{13} \mathrm{C}$ NMR (125 MHz, $\mathrm{CDCl}_{3}$ ) $\delta 135.6$ (2C), 117.5 (2C), 78.3 (2C), 70.1 (2C), $63.4(2 \mathrm{C})$.

General procedure for the preparation of MTPA-esters:

A $0.01 \mathrm{M}$ solution of alcohol product in anhydrous $\mathrm{CH}_{2} \mathrm{Cl}_{2}$ at $0{ }^{\circ} \mathrm{C}$ was treated successively with 2,6-lutidine (8 mol equiv), MTPA-Cl (4 mol equiv per $\mathrm{OH}$ ) and DMAP (1 mol equiv per $\mathrm{OH}$ ) under $\mathrm{N}_{2}$. The mixture was allowed to stir at room temperature for $15 \mathrm{~h}$ and treated with $3(\mathrm{~N}, \mathrm{~N}-$ dimethylamino)propylamine ( $0.3 \mathrm{~mol}$ equiv over acid chloride). After stirring for an additional $10 \mathrm{~min}$, the solvent was removed in vacuo. The residue was dissolved in a minimum amount of $\mathrm{CH}_{2} \mathrm{Cl}_{2}$ and applied to a silica gel column (5\% Et $2 \mathrm{O}$-hexane) to afford pure ester in $85-90 \%$ yield:

(+)-(3R,8S)-1-(S)-MTPA-bis-ester: ${ }^{1} \mathrm{H}$ NMR (500 MHz, $\left.\mathrm{CDCl}_{3}\right)$ 7.52-7.50 (m, 5H), 7.41-7.38 $(\mathrm{m}, 5 \mathrm{H}), 6.40(\mathrm{~d}, \mathrm{~J}=9.0 \mathrm{~Hz}, 1 \mathrm{H}), 6.09(\mathrm{~d}, \mathrm{~J}=5.4 \mathrm{~Hz}, 1 \mathrm{H}), 5.92(\mathrm{~m}, 1 \mathrm{H}), 5.73($ brm, $1 \mathrm{H}), 5.60(\mathrm{~d}$, $\mathrm{J}=16.5 \mathrm{~Hz}, 1 \mathrm{H}), 5.46(\mathrm{~m}, 1 \mathrm{H}), 5.43(\mathrm{~d}, \mathrm{~J}=10.5 \mathrm{~Hz}, 1 \mathrm{H}), 3.63(\mathrm{~s}, 3 \mathrm{H}), 3.58(\mathrm{~s}, 3 \mathrm{H}), 2.15(\mathrm{~m}, 2 \mathrm{H})$, 1.45-1.17 (m, 10H), $0.88(\mathrm{t}, \mathrm{J}=6.9 \mathrm{~Hz}, 1 \mathrm{H}) .(+)-(3 R, 8 S)-1-(R)-M T P A-b i s-e s t e r:{ }^{1} \mathrm{H}$ NMR $(500$ $\left.\mathrm{MHz}, \mathrm{CDCl}_{3}\right) \delta$ 7.52-7.49 (m, 5H), 7.41-7.39 (m, 5H), $6.35(\mathrm{~d}, \mathrm{~J}=8.6 \mathrm{~Hz}, 1 \mathrm{H}), 6.11(\mathrm{~d}, \mathrm{~J}=5.8$ 
$\mathrm{Hz}, 1 \mathrm{H}), 5.82$ (ddd, J= 16.9, 10.2, $5.8 \mathrm{~Hz}, 1 \mathrm{H}), 5.77(\mathrm{~m}, 1 \mathrm{H}), 5.55(\mathrm{~m}, 1 \mathrm{H}), 5.52$ (d, J= 16.9 Hz, $1 \mathrm{H}), 5.37(\mathrm{~d}, \mathrm{~J}=10.2 \mathrm{~Hz}, 1 \mathrm{H}), 3.60(\mathrm{~s}, 3 \mathrm{H}), 3.57(\mathrm{~s}, 3 \mathrm{H}), 2.17(\mathrm{~m}, 2 \mathrm{H}), 1.40-1.24(\mathrm{~m}, 10 \mathrm{H}), 0.87$ (t, J=7.1 Hz, 1H). 


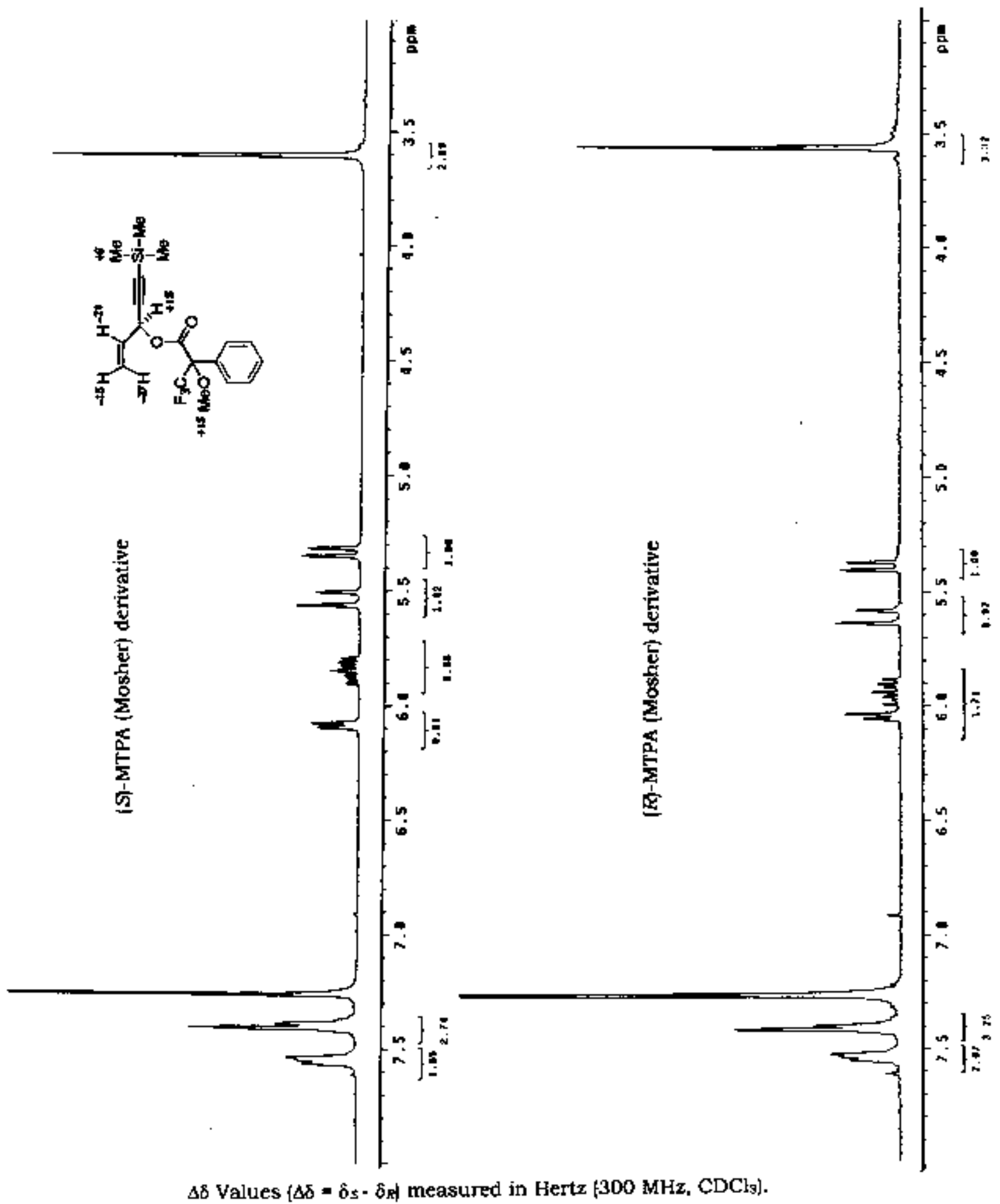


(t)-3-[S-MTPA ester
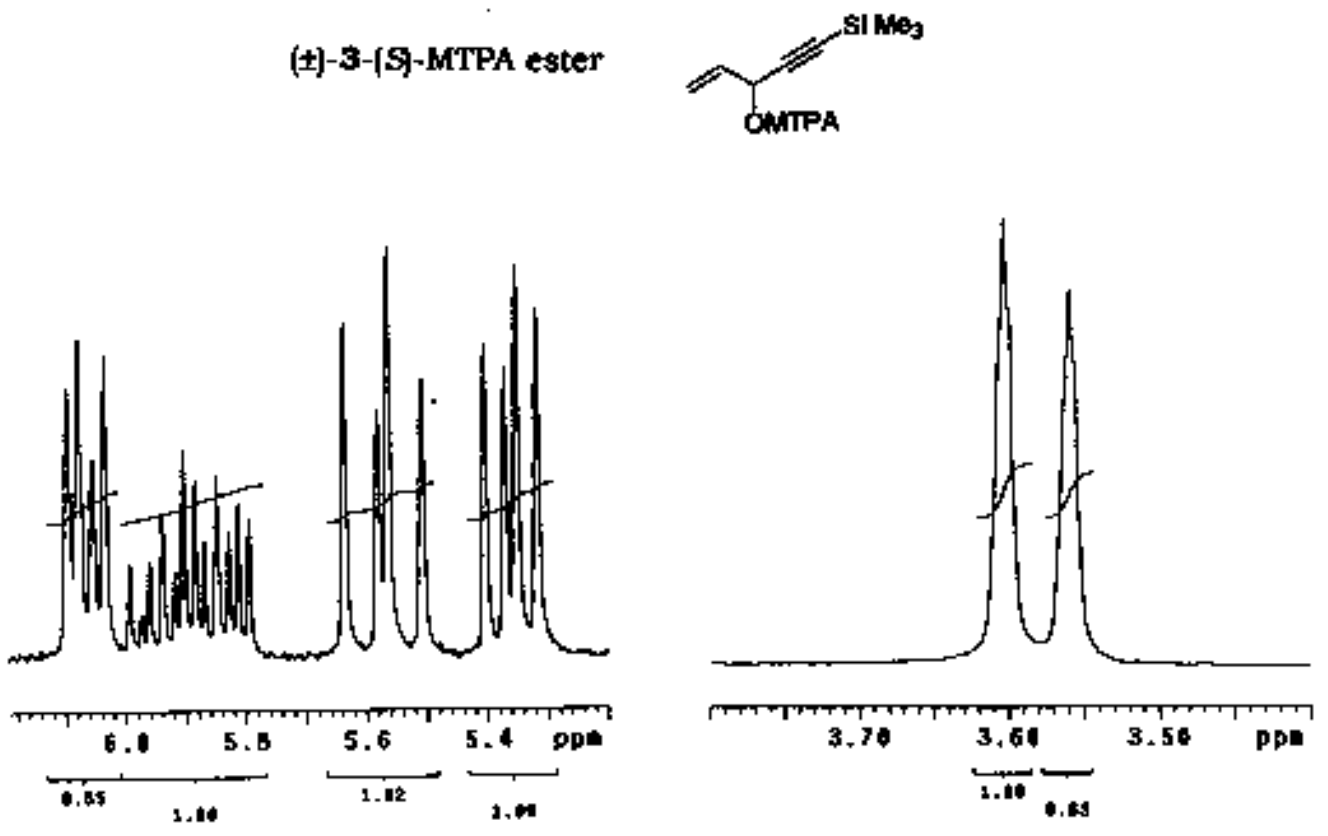

|+1-3-(\$-MTPA ester
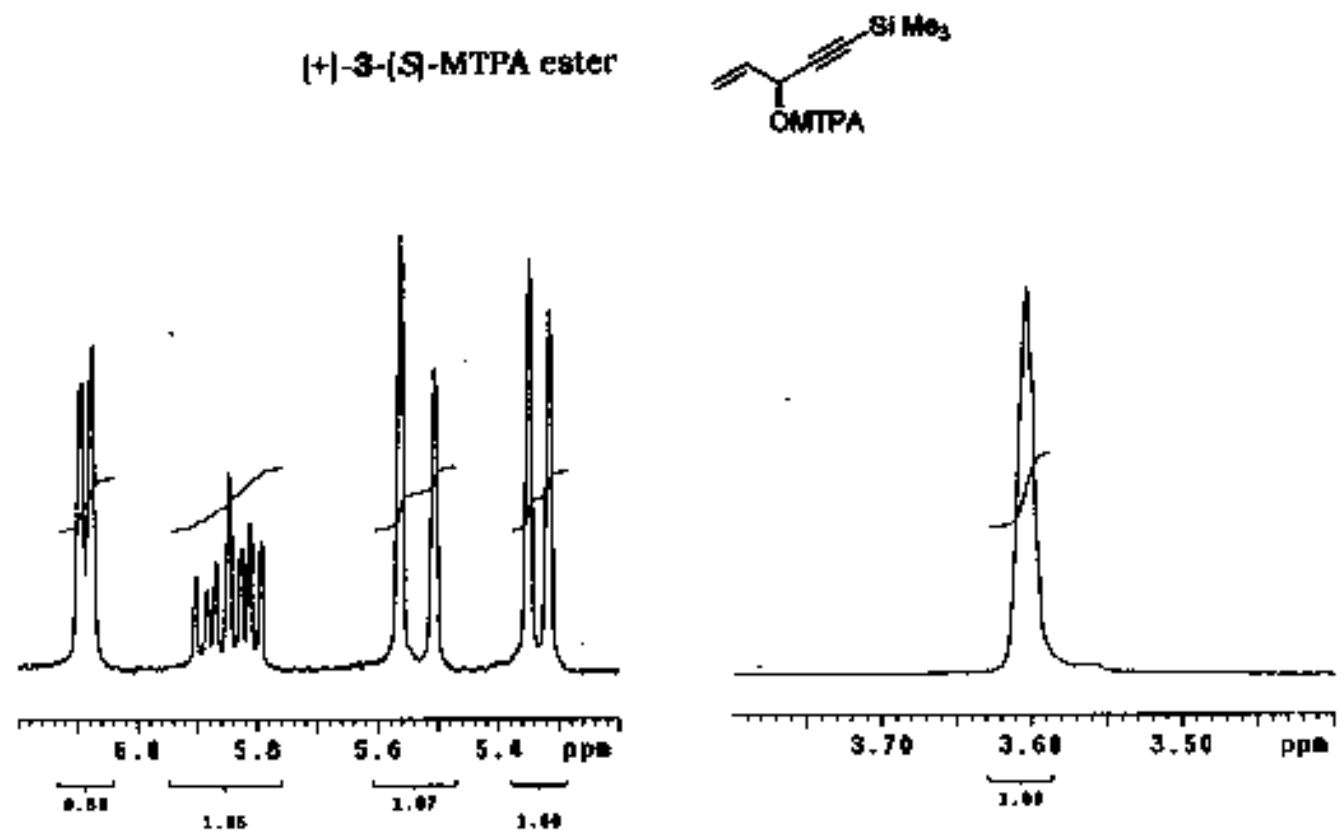

(S)-MTPA (Mosher) derivatives of 3 (300 $\left.\mathrm{MHz}, \mathrm{CDCl}_{3}\right\}$ ). 
2 (meso)

retention time (min)- 16.28 [R,S]

Synthets [t\}-A (racemic) retention times (min)- 13,90 (R,R) $16.27(\mathrm{R}, \mathrm{S})$ $19.33(\mathrm{~S}, \mathrm{~S})$

Synthetic [+]-2 (chira]) retention time (min)- 19.22 (S,S)
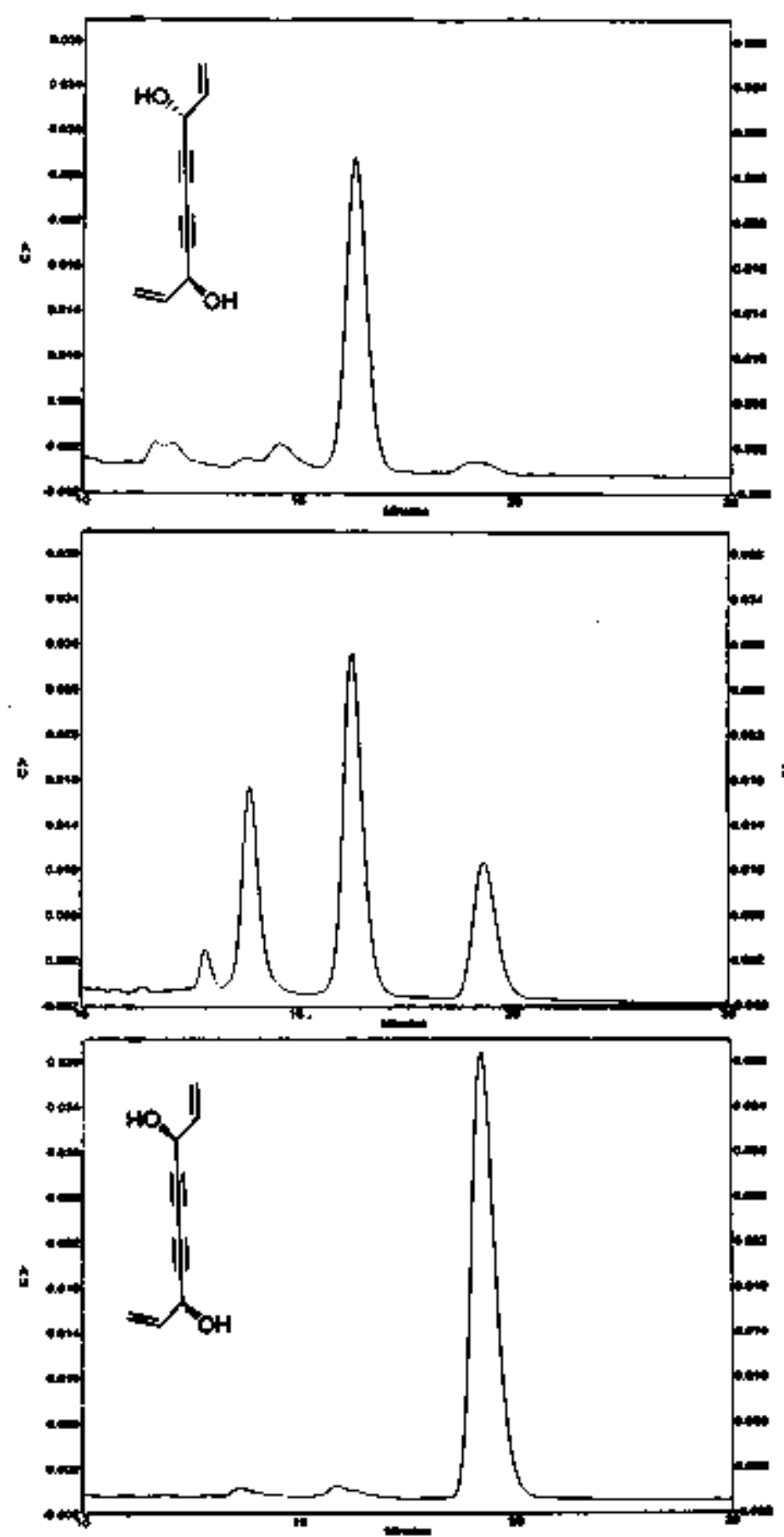

HPLC (chira]) Chiralcel OD [250 $4.6 \mathrm{~mm}), 23^{\circ} \mathrm{C}, \lambda=254 \mathrm{~nm}$, $15 \%$ PrOH-hexane, $1 \mathrm{~mL} / \mathrm{min}$ 